\title{
MicroRNA-125b as a Preeclampsia Biomarker
}

\author{
Bryan Gervais de Liyis ${ }^{1}$, Angela Faustine Ciaves ${ }^{1}$, Elliana Freya Hernowo ${ }^{1}$, \\ Wilson Halim ${ }^{1}$, Desak Made Wihandani \\ ${ }^{1}$ Faculty of Medicine, Udayana University, Denpasar, Bali, Indonesia \\ ${ }^{2}$ Department of Biochemistry, Faculty of Medicine, Udayana University, Denpasar, Bali, Indonesia
}

Corresponding Author: Bryan Gervais de Liyis

\begin{abstract}
Pregnancy hypertensive disorder (PHD) is a broad term referring to conditions related to disorders of high blood pressure during pregnancy. Preeclampsia is one of 4 types of PHD characterized by an increase in systolic blood pressure $\leq 140 \mathrm{mmHg}$ with a diastolic pressure $\leq 90 \mathrm{mmHg}$ accompanied by proteinuria of $1+$ or more. Complications of PHD include peripartum cardiomyopathy, maternal mortality, pulmonary edema, cerebrovascular accident and renal failure. As consequence, early detection of PHD is crucial in preventing complications. "Preeclampsia", "microRNA-125b" and "Biomarker" were the keywords applied to scientific online databases, such as Science Direct, PubMed, and ResearchGate. A total of 46 journals were screened, reviewed and utilized with utmost precision to construct this literature review. Looking at the dangers of preeclampsia on infants and mothers, miRNA-125b has potential to be used as a biomarker of preeclampsia that can occur in the third trimester. The increase in the concentration of miRNA-125b in the first trimester causes inhibition of trophoblast invasion, which is followed by inhibition of KCNA1, GPC1, and SGPL1. All of these proteins play a role in the invasion trophoblast which is also exacerbated by the increase in the concentration of mir-125b. All in all, these symptoms and the increase of mir-125b that appear in the first trimester yield as a promising result as biomarkers of preeclampsia in the third trimester.
\end{abstract}

Key words: preeclampsia, microRNA-125b, biomarker, SGPL1, maternal, infants

\section{INTRODUCTION}

Pregnancy hypertensive disorder is a broad terminology used to refer to conditions related to high blood pressure disorders during the period of pregnancy. ${ }^{[1]}$ Hypertension is the most commonly presented medical disorder observed during periods of pregnancy and complicates up to $15 \%$ of all pregnancies in women of childbearing age, which is a $25 \%$ increase in incidence in the past two decades. ${ }^{[2,3]}$ Furthermore, hypertensive disorders are found to contribute to approximately $7 \%$ to $12 \%$ pregnancy-related maternal deaths. ${ }^{[2]}$ The inclining incidence of pregnancy hypertensive disorder is associated with obesity, advanced maternal age and women who have associated comorbidities. ${ }^{[3]}$ Moreover, women with chronic hypertension are associated with a fivefold higher risk for peripartum cardiomyopathy, maternal mortality, pulmonary edema, cerebrovascular accident and renal failure. ${ }^{[4-}$ ${ }^{6]}$ Furthermore, there are four classification of pregnancy hypertensive disorders namely chronic hypertension, gestational hypertension, preeclampsia and preeclampsia superimposed on chronic hypertension. ${ }^{[7]}$

Preeclampsia is a gestational disorder and multiple systems disorder related with elevation of systolic blood pressure of equal to or greater than 140 $\mathrm{mmHg}$ alongside with a diastolic blood pressure of equal to or greater than 90 $\mathrm{mmHg}$ and accompanied with a proteinuria of $1+$ or more $(0.3 \mathrm{~g}$ per $24 \mathrm{~h}) .{ }^{[8]}$ 
Hypertension and proteinuria during pregnancy coincides with dysfunctions of maternal organs which could be renal insufficiency. ${ }^{[9]}$ Preeclampsia occurs after the 20th week of pregnancy and is estimated as one of the most common complications of pregnancy; affecting 5-10\% of pregnant mothers and accounts for $40 \%$ of fetal deaths worldwide. ${ }^{[8,10]}$ In Indonesia alone, preeclampsia is the third highest leading cause $(13 \%)$ of maternal mortality, after bleeding (45\%) and infections (15\%), with an increasing trend of $40 \%$ per year and $80 \%$ inclining incidence of pre-eclampsia associated-comorbid in pregnant women. ${ }^{[11]}$ It is universally acknowledged that preeclampsia started as asymptomatic, with defects in trophoblast cells invasion as well as spiral artery remodeling in the phase of early pregnancy, which ultimately affects abnormal placentation, entailing to placental ischemia and maternal systemic syndrome in the later phase of gestation. ${ }^{[12]}$ Infants born to preeclamptic mothers were associated with an over fivefold increased risk for infant mortality, a three to sevenfold increased risk for poorer birth outcomes, a three to fivefold increased risk for low birth weight, and compromised neurodevelopmental index. ${ }^{[13]}$

MicroRNAs (miRNAs) are short RNA molecules consisting of 19 to 25 nucleotides in size that control posttranscriptional silencing of the targeting genes. ${ }^{[14]}$ Recent studies suggest that miRNAs are shown to be upregulated in the development of preeclampsia and are suggested to inhibit angiogenesis, trophoblast cells proliferation, placentation and migration. ${ }^{[15]}$ One of the highly upregulated miRNAs, miRNA-125b, could be a promising early non-invasive biomarker. ${ }^{[15-18]}$ Research shows that miRNA-125b is upregulated as early as the $12^{\text {th }}-13^{\text {th }}$ week of gestation in the first trimester. ${ }^{[17]}$ The upregulated miRNA-125b upregulated in preeclampsia is responsible for inhibited trophoblast invasion via Potassium Voltage-gated Channel Subfamily A Member 1 (KCNA1) and transfection of miRNA-125b suppresses endothelial function through Glypican 1 (GPC1). ${ }^{[16]}$ Among these candidate target genes, miRNA-125b agonists is found to reduce both mRNA and protein levels of sphingosine-1-phosphate lyase 1 (SGPL1) in plates of placenta trophoblast cells, thus showing an inverse correlation with miRNA-125b expression. ${ }^{[17]}$ SGPL1 is a key regulator for normal metabolism of lipids. Altered SGPL1 expression results in severe developmental and functional defects in the basal and chorionic plates of trophoblast cells causing the physiological symptoms of preeclampsia. ${ }^{[17]}$ Furthermore, decreased SGPL1 is associated with congenital malfunctions in angiogenesis regulation, vascular maturation, cardiac development and immunity. ${ }^{[17,19]}$ The upregulated mi-RNA-125b in the first trimester presents the clinical symptoms shown in the later trimester of pregnancy. ${ }^{[16]}$

Nowadays, women whom preeclampsia was suspected, a ratio of serum sFlt-1 to PIGF is used as predictive factor of the absence or presence of preeclampsia in the short term. ${ }^{[20]}$ Unfortunately, at one week the positive predictive value was $21 \%$, and at four weeks, it was $18 \% .^{[21]}$ In regards to current biomarker testing, a new biomolecular approach associated with miRNA-125b is suspected to show promising results in diagnosing preeclampsia in the first trimester. As no prior literature review has discussed this before, the authors aim to discuss and evaluate microRNA-125b potential as a biomarker of preeclampsia. In this review, the authors hope to provide a new theoretical basis for the biomarker of preeclampsia.

\section{MATERIALS AND METHODS}

The review method used was by means of literature review. The literature references are of relevant journals from renowned search engines Science Direct, PubMed, and ResearchGate. Keywords such as "Preeclampsia", "microRNA-125b" and "Biomarker" are searched on the search 
engines. The criteria of inclusion were that of all biomarker for preeclampsia and association to miRNA. Preferred studies should be at least 10 years old since the year of publication and no newer studies contradict against the information provided. From 93 journals that were reviewed, 46 were found to be suitable as reference for this paper. The reviewed information evaluated and analyzed for validity, objectivity and reliability is interpreted and compiled into one scientific literature review.

\section{RESULTS AND DISCUSSIONS \\ Pathophysiology of miRNA-125b in Preeclampsia}

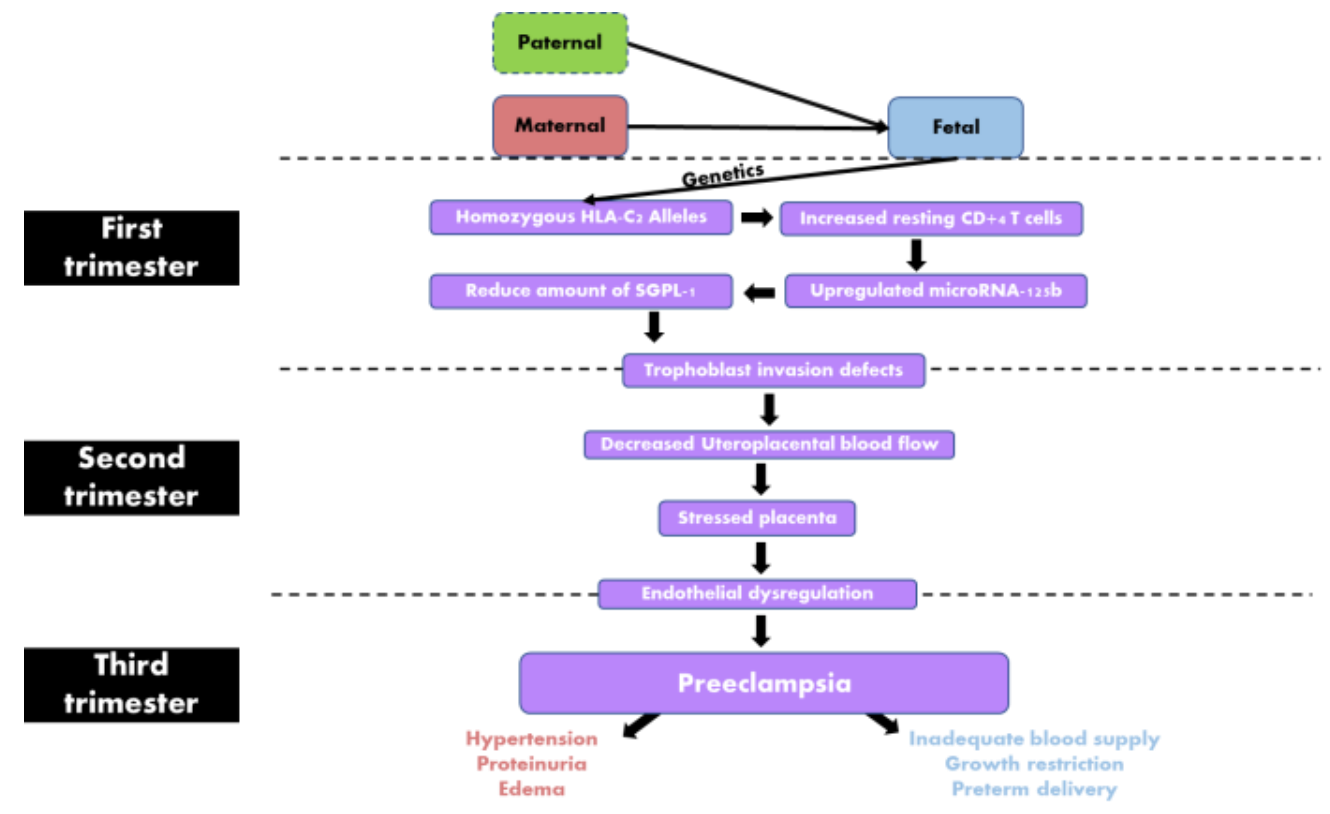

Figure 1. Pathophysiology miRNA-125b induced preeclampsia

As shown in Figure 1, a genetical pathophsiology of preeclampsia is proposed. A study in China revealed that the occurrences of preeclampsia increase only when homozygous HLA-C2 alleles appear in the fetus, indicating that preeclampsia happens when both the paternal and maternal genes carry the HLA-C2 allele. ${ }^{[22]}$ It is also proposed that higher HLA-C expression results in a stronger HLA-Crestricted T-cell response. ${ }^{[23]}$ As a consequence, the resting $\mathrm{CD} 4+\mathrm{T}$ cells enrich expression of miRNA-28, miRNA125b, miRNA-150, miRNA-223, and miRNA-382 in the first trimester. ${ }^{[17,24]}$ One of which, miRNA-125b, is found to reduce both mRNA and protein levels of sphingosine-1-phosphate lyase 1 (SGPL1) in plates of placenta trophoblast cells causing developmental defects. ${ }^{[17]}$ The failure of trophoblast uterine interactions in the first trimester leads to a stress response in the placenta which subsequently affects growth and development of the villous tree, affecting oxygen and nutrients transport to the fetus in the second trimester. ${ }^{[17,25]}$

Stressing of Syncytiotrophoblasts, cells that line the placental villi in contact with maternal blood, releases a complex mix of factors, including pro-inflammatory cytokines, exosomes, anti-angiogenic agents, and cell-free fetal DNA, into the maternal circulation. ${ }^{[26,27]}$ These disrupt maternal endothelial function resulting in a systemic inflammatory response, the clinical syndrome of preeclampsia. ${ }^{[2]}$ Stressed placenta leads a systemic inflammatory response resulting from disruption of the homoeostatic functions of the maternal endothelium, including regulation of clotting, fluid transfer, and blood pressure. ${ }^{[19,25]}$ As consequence, higher levels 
of placental apoptotic debris, placental senescence, cell-free fetal DNA, maternal serum pro inflammatory cytokines, soluble receptor (sFLT) for vascular endothelial growth factor (VEGF), and the lower levels of placental growth factor (PIGF) can be detected. ${ }^{[28-30]}$ Elevated levels of sFlt are suspected to bind and reduce the bioavailability of VEGF towards the maternal endothelial cells, impairing endogenous production of nitric oxide (NO), causing vasoconstriction of blood vessels, and thus hypertension. ${ }^{[31]}$ In terms of endothelium dysregulation, preeclampsia is a global systemic syndrome which can affect many organs such as kidney, liver, nervous system and the coagulation cascade presented as physiological preeclamptic symptoms detected in the third trimester of pregnancy. ${ }^{[9]}$

\section{Role of miRNA in Preeclampsia}

Preeclampsia has complex pathophysiologic mechanisms involved in the process of its pathogenesis. It is a syndrome caused by a variety of factors, mainly including abnormal placental function, immune- system alterations, increased inflammatory activation, abnormal balance of angiogenic and antiangiogenic factors, metabolic changes. ${ }^{[32]}$ Placental dysfunction can result in increased release of extracellular vesiclederived miRNAs, circulating placental factors that have been conserved to contribute to the pathogenesis of preeclampsia. ${ }^{[33,34]}$ Trophoblast cells have been found to have important links to the pathogenesis of preeclampsia through FOXA1 inhibition and the trophoblast cells placental invasion blockade by highly expressed miRNA-20a. ${ }^{[35]}$ In pregnancy hypertensive disorder patients, studies showed a significant increase in miRNA1233 expression that suppresses the expression levels of HoxB3-major regulator of angiogenesis-which leads to proliferation and trophoblast cells invasion decrease in placental tissue from pregnancy hypertensive disorder patient. ${ }^{[35,36]}$ Another three preeclampsia rodent model studies show elevated miR-210 in part driven by HIF- $1 \alpha$ and NF- $\mathrm{Bp} 50$, induced by hypoxia and/or immune-mediated processes may contribute in preeclampsia by inhibiting anti-inflammatory Th2-cytokines. ${ }^{[37]}$

\section{miRNA125b as Biomarker Third Trimester of Pregnancy Hypertensive Disorder}

miR-125b in pregnant women can be detected from several different sites, the plasma from the mother, where it is often called the maternal circulating miR-125b and the placental cells, where it is called as placental miR-125b. ${ }^{[38-40]}$ Circulating miR$125 \mathrm{~b}$ supposedly comes from the placenta itself, as one in vivo study suggests, making the maternal circulating miR-125b and placental miR-125 closely related to one another. ${ }^{[41]}$ Normally, the placental miR$125 \mathrm{~b}$ will experience an eight fold change from the first to the third trimester. ${ }^{[39]}$ In women who went on to develop preeclampsia (PE), one of the subtypes of the hypertensive disorders of pregnancy, several studies exhibit a significant over expression of maternal circulating miR$125 \mathrm{~b}$ by the $12^{\text {th }}$ or $13^{\text {th }}$ week of pregnancy when compared to the healthy control group. ${ }^{[2,16,17,38]}$ Entering the third trimester, PE symptoms have established and the level of circulating miR-125b in women that has developed symptoms of PE is significantly lower than the level of plasma miR-125b found in normal healthy women. ${ }^{[16,40]} \mathrm{miR}$ 125 levels in peripheral whole blood is also down regulated in women with clinically diagnosed PE, gestational hypertension, another subtype of hypertensive disorder of pregnancy (PHD), and intrauterine growth restriction. ${ }^{[0]}$ These findings support that the progression of miR-125 in PE women and normal women were reversed and screening of PE can start as soon as entering the first trimester or around $12^{\text {th }}$ week or $13^{\text {th }}$ week of gestation.

miR-125b over expression in the first trimester on PE women has been discovered to directly cause the down 
regulation of trophoblast cell surface antigen 2 (Trop-2) placental expression, as it is found that miR-125b targets Trop-2 expression in several tissues, including cancer and placenta. ${ }^{[38,42,43]}$ Trop-2, a surface marker for trophoblasts, is also found to be down regulated in hypoxic conditions, a common condition in $\mathrm{PE}$ women. ${ }^{[38,44,45]}$ miR-125b also directly targets the KCNA1 glycoprotein, usually found on trophoblast cells, and GPC1 on vascular endothelial cells, with higher levels of $\mathrm{miR}-125 \mathrm{~b}$ in the first trimester is associated with a decreasing KCNA1 and GPC1. ${ }^{[16]}$ As both Trop-2 and KCNA1 are found in trophoblast cells, it is concluded that miR-125b can inhibit trophoblast invasion from the first trimester, long before symptoms manifest, and may lead to the development of PE, which is characterized by hypoxic conditions in the placenta, thus further down regulate the expression of Trop2. ${ }^{[16,38,46]}$ In the same study, it was also discovered that miR-125b is directly involved in tube formation in human umbilical vascular endothelial cells or HUVECs, with administration of GPC1 can reverse the effect caused by miR-125b, and this may present as a sign leading to endothelial dysfunction caused by an over expression of miR-125b in the first trimester. ${ }^{[16,46]}$ The role of miR-125b in IL-8 up regulations has been observed through the interaction of SGPL-1 and miR-125b, as an up regulated level of miR-125 will decrease the number of SGPL- 1 and in turn increase the level of IL-8, a commonly found inflammation cytokine in PE and thought to be one of the major factors on PE development in the first trimester. ${ }^{[17]}$

\section{Potential miRNA-125b Inhibitor as Prevention in Third Trimester Pregnancy Hypertensive Disorder}

Research by Licini et al. using a placenta sample in the first trimester $\left(12^{\text {th }}\right.$ week gestational age) showed decreased Trop-2 expression in preeclampsia compared to normal pregnancy. ${ }^{[38]}$ This was supported by overexpression of miR-125b in the first trimester and underexpression of miR-125b in the third trimester of placental $\mathrm{PE}$ with a significant reduction compared to placental CTR. ${ }^{[38-40]}$ Besides, over expression of miR-125b in the first trimester led to inhibition of KCNA1 and GPC1 expression. The level of KCNA1 and GPC1 mRNA significantly decreased as much as \pm $51 \%$ and $\pm 35 \%$ and as much as $30 \%$ and $50 \%$ on the decreased levels of KCNA1 and GPC1 proteins. $^{[16]}$ In the same study, a significant increase in miR-125b expression led to $58.6 \%$ inhibition of trophoblast invasion. Experiments using the miR-125b inhibitor significantly counteracted the inhibitory effect of miR-125b invasion. Also, inhibition of trophoblast invasion was associated with decreased KCNA1 by over expression of miR-125b. ${ }^{[16]}$ The inhibitory effect of miR-125b was also inhibited by elevated GCP1. Both of these were evidenced by the addition of KCNA1 and GCP1 levels in the test sample. ${ }^{[16]}$ Research that has been conducted by Yang et al. using the human trophoblast cell line, HTR8/SVneo cells, found that SGPL1 levels decreased by overexpression of miR$125 \mathrm{~b}$. Test with the addition of miR-125 inhibitor can increase SGPL1 levels which can improve trophoblast invasion in placenta PE. ${ }^{[17]}$

In conclusion, miRNAs that play a role in the incidence rate of preeclampsia. According to research, miRNA-125b is often causing preeclampsia by inhibiting trophoblast invasion in suppression of KCNA1, GPC1, and SGPL1. The potential of using miRNA-125b as a biomarker of preeclampsia can be maximized, considering that the pathogenesis of miRNA that causes preeclampsia varies greatly in each individual. Research to date that has been carried out in vivo strongly supports the increase in miRNA-125b concentrations as a cause of preeclampsia.

\section{Acknowledgement: None}

Conflict of Interest: None 


\section{Source of Funding: None}

\section{REFERENCES}

1. Scott G, Gillon TE, Pels A, von Dadelszen P, Magee LA. Guidelines-similarities and dissimilarities: a systematic review of international clinical practice guidelines for pregnancy hypertension. Am J Obstet Gynecol [Internet] 2020;Available from: https://www.sciencedirect.com/science/artic le/pii/S0002937820308462

2. Shah S, Gupta A. Hypertensive Disorders of Pregnancy. Cardiol Clin [Internet] 2019;37(3):345-54. Available from: https://doi.org/10.1016/j.ccl.2019.04.008

3. Folk DM. Hypertensive Disorders of Pregnancy: Overview and Current Recommendations. J Midwifery Women's Heal 2018;63(3):289-300.

4. Battarbee AN, Sinkey RG, Harper LM, Oparil S, Tita ATN. Chronic hypertension in pregnancy. Am J Obstet Gynecol [Internet] 2020;222(6):532-41. Available from: https://doi.org/10.1016/j.ajog.2019.11.1243

5. Orabona R, Sciatti E, Prefumo F, Vizzardi E, Bonadei I, Valcamonico A, et al. Preeclampsia and heart failure: a close relationship. Ultrasound Obstet Gynecol 2018;52(3):297-301.

6. C. ME. Preeclampsia and Cerebrovascular Disease. Hypertension [Internet] 2019;74(1):5-13. Available from: https://doi.org/10.1161/HYPERTENSIONA HA. 118.11513

7. Dines V, Kattah A. Hypertensive Disorders of Pregnancy. Adv Chronic Kidney Dis [Internet] 2020;27(6):531-9. Available from:

https://doi.org/10.1053/j.ackd.2020.05.006

8. Rahnemaei FA, Fashami MA, Abdi F, Abbasi M. Factors effective in the prevention of Preeclampsia:A systematic review. Taiwan J Obstet Gynecol [Internet] 2020;59(2):173-82. Available from: https://doi.org/10.1016/j.tjog.2020.01.002

9. Mayrink J, Costa ML, Cecatti JG. Preeclampsia in 2018: Revisiting Concepts, Physiopathology, and Prediction. Sci World J 2018;2018.

10. Benny PA, Alakwaa FM, Schlueter RJ, Lassiter CB, Garmire LX. A review of omics approaches to study preeclampsia. Placenta [Internet] 2020;92(May 2019):17-
27. Available from: https://doi.org/10.1016/j.placenta.2020.01.0 08

11. Setyorini D, Santoso B, Martini S, Ernawati. Risk Factors of Preeclampsia and Eclampsia in Surabaya. Dama Int J Res 2017;2(7):636.

12. Rana S, Lemoine E, Granger J, Karumanchi SA. Preeclampsia: Pathophysiology, Challenges, and Perspectives. Circ Res 2019;124(7):1094-112.

13. Nomura Y, Finik J, Salzbank J, Ly J, Huynh $\mathrm{N}$, Davey $\mathrm{T}$, et al. The Effects of Preeclampsia on Perinatal Risks and Infant Temperaments Among Mothers With Antenatal Depression. Psychol Res (Libertyville, Ill) [Internet] 2014;4(6):45161. Available from: https://pubmed.ncbi.nlm.nih.gov/30009089

14. Lu TX, Rothenberg ME. MicroRNA. J Allergy Clin Immunol 2018;141(4):1202-7.

15. Rudov A, Balduini W, Carloni S, Perrone S, Buonocore G, Albertini MC. Involvement of miRNAs in placental alterations mediated by oxidative stress. Oxid Med Cell Longev 2014;2014.

16. Li Q, Han Y, Xu P, Yin L, Si Y, Zhang C, et al. Elevated microRNA-125b inhibits cytotrophoblast invasion and impairs endothelial cell function in preeclampsia. Cell Death Discov [Internet] 2020;6(1). Available from: http://dx.doi.org/10.1038/s41420-020-02690

17. Yang W, Wang A, Zhao C, Li Q, Pan Z, Han X, et al. MiR-125b enhances IL-8 production in early-onset severe preeclampsia by targeting sphingosine-1phosphate lyase 1. PLoS One 2016;11(12):1-17.

18. Gasparri ML, Casorelli A, Bardhi E, Besharat AR, Savone D, Ruscito I, et al. Beyond circulating microRNA biomarkers: Urinary microRNAs in ovarian and breast cancer. Tumor Biol 2017;39(5).

19. Janecke AR, Xu R, Steichen-Gersdorf E, Waldegger S, Entenmann A, Giner T, et al. Deficiency of the sphingosine-1-phosphate lyase SGPL1 is associated with congenital nephrotic syndrome and congenital adrenal calcifications. Hum Mutat [Internet] 2017;38(4):365-72. Available from: https://pubmed.ncbi.nlm.nih.gov/28181337

20. Zeisler H, Llurba E, Chantraine F, Vatish M, Staff AC, Sennström M, et al. Predictive 
Value of the sFlt-1:PlGF Ratio in Women with Suspected Preeclampsia. N Engl J Med [Internet] 2016;374(1):13-22. Available from:

https://doi.org/10.1056/NEJMoa1414838

21. Caillon H, Tardif C, Dumontet E, Winer N, Masson D. Evaluation of sFlt-1/PIGF Ratio for Predicting and Improving Clinical Management of Pre-eclampsia: Experience in a Specialized Perinatal Care Center. Ann Lab Med [Internet] 2018;38(2):95-101. Available from: https://pubmed.ncbi.nlm.nih.gov/29214752

22. Long W, Shi Z, Fan S, Liu L, Lu Y, Guo X, et al. Association of maternal KIR and fetal HLA-C genes with the risk of preeclampsia in the Chinese Han population. Placenta 2015;36(4):433-7.

23. Blais ME, Dong T, Rowland-Jones S. HLA$\mathrm{C}$ as a mediator of natural killer and T-cell activation: Spectator or key player? Immunology 2011;133(1):1-7.

24. Gibson DA, Simitsidellis I, Collins F, Saunders PTK. Endometrial intracrinology: Oestrogens, androgens and endometrial disorders. Int. J. Mol. Sci.2018;19(10).

25. Burton GJ, Redman CW, Roberts JM, Moffett A. Pre-eclampsia: pathophysiology and clinical implications. BMJ [Internet] 2019;366. Available from: https://www.bmj.com/content/366/bmj.1238 1

26. Tannetta D, Masliukaite I, Vatish M, Redman C, Sargent I. Update of syncytiotrophoblast derived extracellular vesicles in normal pregnancy and preeclampsia. J Reprod Immunol 2017; 119:98-106.

27. Redman CW, Sargent IL, Staff AC. IFPA Senior Award Lecture: making sense of preeclampsia - two placental causes of preeclampsia? Placenta 2014;35 Suppl:S205.

28. Mizuuchi M, Cindrova-Davies T, Olovsson M, Charnock-Jones DS, Burton GJ, Yung HW. Placental endoplasmic reticulum stress negatively regulates transcription of placental growth factor via ATF4 and ATF6 $\beta$ : Implications for the pathophysiology of human pregnancy complications. J Pathol 2016;238(4):55061.

29. Sekizawa A, Jimbo M, Saito H, Iwasaki M, Matsuoka R, Okai T, et al. Cell-free fetal DNA in the plasma of pregnant women with severe fetal growth restriction. Am J Obstet Gynecol 2003;188(2):480-4.

30. Cindrova-Davies T, Fogarty NME, Jones CJP, Kingdom J, Burton GJ. Evidence of oxidative stress-induced senescence in mature, post-mature and pathological human placentas. Placenta 2018;68:15-22.

31. 31. Cindrova-Davies T, Sanders DA, Burton GJ, Charnock-Jones DS. Soluble FLT1 sensitizes endothelial cells to inflammatory cytokines by antagonizing VEGF receptor-mediated signalling. Cardiovasc Res 2011;89(3):671-9.

32. Lv Y, Lu C, Ji X, Miao Z, Long W, Ding H, et al. Roles of microRNAs in preeclampsia. J Cell Physiol 2019;234(2):1052-61.

33. Biró O, Alasztics B, Molvarec A, Joó J, Nagy B, Rigó J. Various levels of circulating exosomal total-miRNA and miR210 hypoxamiR in different forms of pregnancy hypertension. Pregnancy Hypertens [Internet] 2017;10(April):20712. Available from: http://dx.doi.org/10.1016/j.preghy.2017.09.0 02

34. Murakami Y, Miura K, Sato S, Higashijima A, Hasegawa Y, Miura S, et al. Reference values for circulating pregnancy-associated microRNAs in maternal plasma and their clinical usefulness in uncomplicated pregnancy and hypertensive disorder of pregnancy. J Obstet Gynaecol Res 2018;44(5):840-51.

35. Zhong W, Peng H, Tian A, Wei Y, Li H, Tian J, et al. Expression of miRNA-1233 in placenta from patients with hypertensive disorder complicating pregnancy and its role in disease pathogenesis. Int J Clin Exp Med 2015;8(6):9121-7.

36. Zhou J, Guo X, Sun Y, Ma L, Zhe R. Levels of serum Hoxb3 and sFlt-1 in pre-eclamptic patients and their effects on pregnancy outcomes. J Obstet Gynaecol Res 2020;46(10):2010-8.

37. Frazier S, McBride MW, Mulvana $H$, Graham D. From animal models to patients: The role of placental microRNAs, miR-210, miR-126, and miR-148a/152 in preeclampsia. Clin Sci 2020;134(8):100125.

38. Licini C, Avellini C, Picchiassi E, Mensà E, Fantone S, Ramini D, et al. Pre-eclampsia predictive ability of maternal miR-125b: a clinical and experimental study. Transl Res 2021;228:13-27. 
39. Gu Y, Sun J, Groome LJ, Wang Y. Differential miRNA expression profiles between the first and third trimester human placentas. Am J Physiol - Endocrinol Metab 2013;304(8):836-43.

40. Hromadnikova I, Kotlabova K, Hympanova L, Krofta L. Gestational hypertension, preeclampsia and intrauterine growth restriction induce dysregulation of cardiovascular and cerebrovascular disease associated microRNAs in maternal whole peripheral blood. Thromb Res [Internet] 2016;137:126-40. Available from: http://dx.doi.org/10.1016/j.thromres.2015.11 .032

41. Reliszko ZP, Gajewski Z, Kaczmarek MM. Signs of embryo-maternal communication: miRNAs in the maternal serum of pregnant pigs. Reproduction 2017;154(3):217-28.

42. Nakanishi H, Taccioli C, Palatini J, Fernandez-Cymering C, Cui R, Kim T, et al. Loss of miR-125b-1 contributes to head and neck cancer development by dysregulating TACSTD2 and MAPK pathway. Oncogene 2014;
43. Avellini C, Licini C, Lazzarini R, Gesuita R, Guerra E, Tossetta G, et al. The trophoblast cell surface antigen 2 and miR$125 \mathrm{~b}$ axis in urothelial bladder cancer. Oncotarget 2017;

44. Lenárt S, Lenárt P, Šmarda J, Remšík J, Souček K, Beneš P. Trop2: Jack of all trades, master of none. Cancers (Basel) 2020;12(11):1-28.

45. Mcdougall ARA, Tolcos M, Hooper SB, Cole TJ, Wallace MJ. Trop2: From development to disease. Dev Dyn 2015;244(2):99-109.

46. Li JYZ, Yong TY, Michael MZ, Gleadle JM. MicroRNAs: Are they the missing link between hypoxia and pre-eclampsia? Hypertens Pregnancy 2014;33(1):102-14.

How to cite this article: Bryan Gervais de Liyis, Ciaves AF, Hernowo EF et.al. MicroRNA-125b as a preeclampsia biomarker. International Journal of Research and Review. 2021; 8(8): 628-635. DOI: https://doi.org/10.52403/ijrr. 20210883 